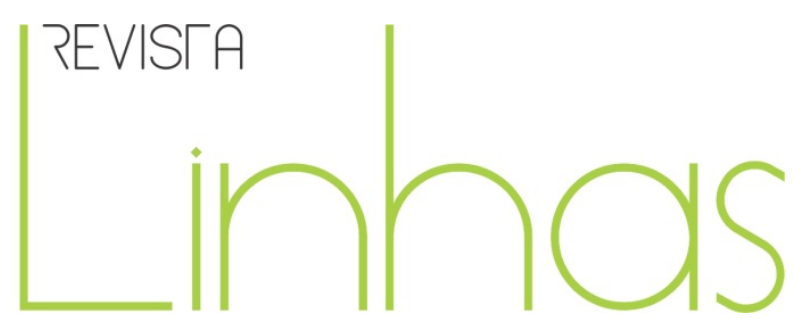

\title{
Les inégalités au Brésil et dans d'autres États émergents. Comparaisons avec un groupe témoin
}

\begin{abstract}
Résumé
Dans cet article, nous mettons en évidence le rôle excessif et nocif que les inégalités exercent même quand il s'agit d'un développement qui respecte, bien que minimalement, les humains et leur environnement planétaire. Le défi auquel nous nous sommes consacrés consiste à analyser, comparativement, les pays relativement nouveaux qui intègrent le BRICS (Afrique du Sud, Brésil, Chine, Inde et Russie) avec un groupe de contrôle, qui comprend l'Allemagne, le Canada, Cuba, les États-Unis et le Japon (quatre d'entre eux appartenant à des pays du G7). Pour ce faire, nous prenons comme référence un ensemble de données sur les revenus, la santé et l'éducation, recueillies par le Programme des Nations Unies pour le développement(PNUD), en particulier l'indice de développement humain (IDH) proposé par le prix Nobel d'économie Amartya Sen. Nos conclusions montrent qu'aujourd'hui, plus qu'au moment de son élaboration il y a un demi-siècle, les théories de l'héritage culturel et de la reproduction sociale de Pierre Bourdieu et Jean-Claude Passeron (1964 et 1970) sont toujours d'actualité et le resteront tant que les inégalités ne sont pas éradiquées.
\end{abstract}

Mots-clés: Développement humain; Revenu; Santé; Éducation; Héritage culturel; Reproduction sociale.

\section{Para citar este artigo:}

LANGOUËT, Gabriel. Les inégalités au Brésil et dans d'autres États émergents. Comparaisons avec un groupe témoin. Revista Linhas. Florianópolis, v. 15, n. 29, p. 112-136, jul./dez. 2014. 


\section{Inequalities in Brazil and in other emerging States. Comparisons with a control group}

\begin{abstract}
In this article we seek to highlight the excessive and harmful role that inequalities exert when it comes to a development that should respect, although minimally, humans and their environment, in the totality of the planet. The challenge we set ourselves was to analyze comparatively emerging countries that make up the BRICS (South Africa, Brazil, China, India and Russia) with a control group, embracing Germany, Canada, Cuba, United States and Japan (four of them belonging to the $\mathrm{G} 7$ ). For that, we take as reference a set of data concerning income, health and education, collected by the United Nations Development Program (UNDP), particularly the human development index (HDI) adjusted by the Nobel Prize in Economics Amartya Sen. Our conclusions indicated that, today, more than at the time they were developed, half a century ago, the theories of cultural heritage and social reproduction of Pierre Bourdieu and Jean-Claude Passeron (1964 and 1970) are still relevant and so they will be while inequalities are not undone.
\end{abstract}

Keywords: Human Development; Income; Health; Education; Cultural Inheritance and Social Reproduction.

\section{As desigualdades no Brasil e em outros Estados emergentes. Comparações com um grupo de controle}

\section{Resumo}

Neste artigo procuramos evidenciar o papel excessivo e nocivo que as desigualdades exercem quando se trata de um desenvolvimento que respeite, ainda que minimamente, os humanos e seu meio ambiente, na totalidade do planeta. $O$ desafio a que nos propusemos consistiu em analisar, comparativamente, países emergentes que integram o BRICS (África do Sul, Brasil, China, Índia e Rússia) com um grupo de controle, abrangendo Alemanha, Canadá, Cuba, Estados Unidos e Japão (quatro deles pertencentes ao G7). Para tanto, tomamos como referência um conjunto de dados relativos à renda, saúde e educação, coletados pelo Programa das Nações Unidas para o Desenvolvimento (PNUD), particularmente 0 índice de desenvolvimento humano (IDH) ajustado pelo prêmio Nobel de Economia Amartya Sen. Nossas conclusões indicaram que, hoje, mais do que no momento em que fora elaborada, há meio século, as teorias da herança cultural e da reprodução social de Pierre Bourdieu e Jean-Claude Passeron (1964 e 1970) continuam pertinentes e assim o serão enquanto as desigualdades não forem combatidas.

Palavras-chave: Desenvolvimento humano; renda; saúde; educação; herança cultural e reprodução social. 


\section{Avant-propos}

Cet article se situe dans le prolongement de deux ouvrages ${ }^{1}$ qui s'était attachés à la mise en évidence du rôle excessif et nocif que jouent les inégalités vis-à-vis d'un développement humain moins indécemment réparti, et qui serait respectueux, fut-ce $a$ minima, de l'environnement, sur l'ensemble de la planète . Rappelons-en quelques aspects essentiels, qui éclairent la démarche retenue dans cette nouvelle étude.

Le premier proposait, au niveau de la quasi-totalité des États de la planète - 182, sur 192 ont été observés -, une étude approfondie quant à la manière dont ces inégalités, tant entre les États qu'à l'intérieur des États, s'élaborent, s'entrecroisent et se surajoutent ou se multiplient, notamment en fonction des trois secteurs qui les engendrent principalement : indicateurs économiques estimés notamment à partir du revenu national brut par habitant (RNB/h); indicateurs de santé notamment résumés par l'espérance de vie à la naissance; indicateurs d'éducation résumant d'une part les taux moyens d'alphabétisation des adultes et, d'autre part, le nombre moyen d'années d'espérance de scolarisation des jeunes. Mais aussi d'autres indicateurs sur lesquels nous reviendrons. Une illustration de la complexité des faits sociaux que souligne notamment Edgar Morin, les causes pouvant à leur tour devenir effets, et réciproquement.

La première condition de réalisation de ce travail reposait sur l'obtention de données fiables et homogènes. D’où notre choix porté sur les données brutes collectées par le Programme des Nations-Unies pour le développement (PNUD), pour l'essentiel en 2008, et celui d'utiliser les puissants indices que les équipes du PNUD ont mis au point, notamment l'indice de développement humain mis au point par le prix Nobel d'économie Amartya Sen, et en y adjoignant des indicateurs de répartitions des revenus dans les populations, le coefficient de Gini et les écarts entre les déciles extrêmes.

Que ce soit entre les États ou entre les populations d'un même État, la mesure des inégalités a mis en évidence des écarts d'une ampleur parfois totalement démesurée et intolérable : « trop, c'est trop ! ». Rappelons quelques résultats.

\footnotetext{
${ }^{1}$ LANGOUËT, G. (Préface de R. ESTABLET), Les inégalités entre États et populations de la planète, Trop, c'est trop! , Paris, L'Harmattan, 2011 ; LANGOUËT, G., Les inégalités dans l'Union européenne et ailleurs. Et si on osait?, Paris, L'Harmattan, 2014.
} 
- Revenu national annuel brut par habitant : 140\$ au Burundi, $87000 \$$ en Norvège.

- $\quad$ Espérance de vie : 45 ans en Afghanistan, 83 ans au Japon.

- Mortalité infantile : 150 \% en Afghanistan, $3 \%$ en Suède.

- $\quad$ Nombre d'enfants par femme : 7 au Niger, moins de 2 dans la quasi totalité des États les plus riches.

- $\quad$ Alphabétisation : $26 \%$ des adultes, et $18 \%$ des femmes, au Mali.

- $\quad$ Scolarité primaire achevé : moins d'un enfant sur 3, et encore moins de filles, achève sa scolarité en primaire au Tchad ou en République centrafricaine. Accès à I'enseignement supérieur : $90 \%$ en Finlande

- Rapport de répartition des revenus entre les $10 \%$ les plus riches et les $10 \%$ les plus pauvres : Suède, 6 ; Namibie : $130 \ldots$

Notre second ouvrage s'est attaché à l'étude des inégalités dans l'ensemble des États de I'Union européenne (UE), zone ou hors zone Euro, en comparaison avec un échantillon raisonné de 10 autres États de la planète (dont le Brésil). Selon notre hypothèse principale, issue des observations menées lors de la préparation du précédent, I'UE, tant entre ses États qu'à l'intérieur de ceux-ci, était certes marquée par des inégalités encore trop fortes, mais sans commune mesure avec les inégalités constatées au niveau planétaire. D'où un double objectif : d'abord mieux faire connaître cette réalité ; et surtout montrer que ces proximités, même relatives, pourraient constituer un tremplin, vers de nouvelles avancées ${ }^{2}$.

Le passage par le PNUD s'est à nouveau imposé, tant en ce qui concerne les données qu'il regroupe et actualise de façon permanente (données 2011, cette fois), que pour le concept de développement humain qu'il développe et enrichit. De surcroît, entre 2008 et 2011, il a approfondi ses outils d'analyse du développement humain (en y adjoignant notamment un indice « ajusté aux inégalités ») et élaboré un excellent indice

\footnotetext{
2 Nous en conseillons la lecture, même rapide, car elle prépare, quant aux contenus et méthodologiquement, celle de cet article.
} 
des inégalités de genre. Bref, nous disposions d'un matériau de qualité, et dont la consultation est remarquablement facilitée.

L'accueil qu'il a reçu, malgré un climat politique peu propice (l'engouement des Européens pour I'UE reste mitigé), donne à penser que notre double objectif, d'information et de propositions, a été atteint :

I'UE connaît certes des inégalités, mais des inégalités assez modérées, notamment par rapport à celles observées dans le groupe témoin ;

sur les trois dimensions du développement humain - revenus, vie et santé, éducation -, l'homogénéité domine, facilitant des rapprochements, même si les inégalités selon le genre restent souvent tenaces. Et les écarts apparaissent plutôt moindres, notamment en termes de revenus, que ceux qui étaient observés entre la République fédérale allemande (RFA) et la République démocratique allemande (RDA) avant la réunification ;

$>\quad$ d'autres exemples, notamment ceux de la Finlande ou de la Suède, mais aussi celui de la République tchèque, en particulier en éducation, montrent que la réduction des inégalités, sous toutes leurs formes, n'a pas été un frein au développement. «Et si on osait ? » avons-nous, cette fois, sous titré.

\section{Introduction}

Les recherches brièvement évoquées en avant-propos montrent bien que l'enjeu majeur du 21e siècle, pour l'ensemble des êtres humains comme pour notre planète, qui poursuit son auto-destruction, ce sera celui de la progression du développement humain (Encadré $n^{0} 1$ ), pour tous et, a minima, pour le plus grand nombre ; mais elles mettent aussi en évidence que ce but ne peut être atteint sans nette réduction des inégalités les plus insupportables, entre les États comme à l'intérieur des populations des États. L'idée selon laquelle il suffirait de produire plus pour que la richesse, d'elle-même et sans contrainte, se répartisse n'est qu'une idée fausse, totalement contredite par les faits eux- 
mêmes et la dernière crise mondiale. Elle est néanmoins répétée par les tenants du néolibéralisme mais aussi fermement combattue par de grands économistes de notoriété internationale, qui établissent la nécessité de régulations, en particulier en ce qui concerne la grande finance et les paradis fiscaux. De surcroît, dans un contexte mondialisé, un État seul n'a que peu de poids, sauf s’il figure parmi les très " grands », car il est soumis à des contraintes internationales fortes; et un repli sur des frontières nationales et une économie fermée est sans doute un leurre: c'est plutôt vers une évolution profonde de la mondialisation qu'il faudrait se diriger. Et c"est dans le prolongement de ces travaux antérieurs, et dans le même contexte, que se situe la présente contribution, centrée sur les États dits émergents

Le concept d'émergence économique date du début des années 1980. Les économistes s'accordent pour la plupart sur le fait que les habitants des pays émergents disposent de revenus intermédiaires, compris entre 10 et 75 \% des revenus par habitants de l'Union européenne, et connaissent une croissance économique supérieure à la croissance mondiale moyenne ; ces pays doivent en outre développer une politique économique d'ouverture, donc jouer un rôle significatif dans les échanges mondiaux, d'où l'importance des exportations. De fait, sont surtout exclus les États très pauvres et/ou de populations réduites. Au début des années 2010, environ 60 États (sur près de 200), représentant près de la moitié de la richesse annuelle créée sur la planète et les deux tiers de sa population, répondaient à ces critères. La liste est nécessairement provisoire, certains États rejoignant assez rapidement le groupe des pays développés, d'autres perdant au contraire ce statut intermédiaire, notamment si leur niveau de croissance s'est fortement dégradé.

Face aux regroupements des États les plus riches (le G7 par exemple ${ }^{3}$ ), et afin de pouvoir mieux infléchir les politiques économiques et financières menées dans le cadre de la mondialisation, des États émergents se sont aussi rapprochés. C'est notamment le cas du BRICS ${ }^{4}$. A titre de repère, la planète compte actuellement environ 7 milliards d'humains, et devrait en compter 10 milliards vers 2050. Aujourd'hui, les 5 États

\footnotetext{
${ }^{3}$ Allemagne, Canada, États-Unis, France, Italie, Japon, Royaume-Uni.

${ }^{4}$ Brésil, Russie, Inde, Chine, Afrique du Sud.
} 
émergents regroupés dans le BRICS rassemblent, à eux seuls, près de 3 milliards d'êtres humains soit environ $40 \%$ des 7 milliards ; ils ont contribué pour $27 \%$ au PIB mondial en 2011, mais leur contribution devrait, selon les estimations, s'élever à $40 \%$ dès 2025. Le G7 par exemple, regroupe des États développés, qui au total, n'en comptent qu'un peu plus de 700 millions, soit un peu plus de 10 \% de la population mondiale ; mais, en 2012, sa contribution au PIB mondial était de l'ordre de $47 \% 5$.

Comme nous l'avons fait à propos de I'Union européenne, il a semblé indispensable de constituer un groupe témoin constitué d'États pouvant être comparés à ceux qui composent le BRICS.

Comme le BRICS, il est donc composé de 5 États, et notamment d'États développés aussi représentatifs que possible des grandes puissances économiques de la planète ${ }^{6} .4$ ont été choisis parmi les États du G7, dont 2 sans aucune hésitation : les ÉtatsUnis, première puissance économique mondiale, et le Japon, troisième derrière la Chine et première puissance de l'Asie $;$ le $3^{e}$ État retenu est l'Allemagne, 4e puissance mondiale et pays le plus peuplé de l'UE. Le $4^{\mathrm{e}}$, le Canada, peut davantage se discuter : il n'est, si l'on peut dire, que $8^{\mathrm{e}}$ puissance économique mondiale et, surtout, sa population es réduite par rapport aux autres ; mais ses différences avec les États-Unis méritent observation. Le $5^{\mathrm{e}}$, Cuba, dont le revenu par habitant se situe entre ceux de la Chine et de l'Inde, a été retenu parce qu'il figurerait parmi les grands émergents s'il avait la taille de leurs populations ; et ses résultats, tant dans le domaine de la vie et de la santé que dans celui de l'éducation, semblent à observer en comparaison.

\begin{tabular}{ll}
\hline \multicolumn{1}{c}{$\quad$ États émergents (BRICS) } & Groupe témoin \\
Afrique du Sud & Allemagne \\
Brésil & Canada \\
Chine & Cuba \\
Inde & États-Unis \\
Russie & Japon \\
\hline \multicolumn{2}{c}{ Tableau 1. Les 2 groupes d'États } \\
\hline
\end{tabular}

\footnotetext{
${ }^{5}$ Tous les experts sérieux considèrent que la croissance, si elle se poursuivait à ce rythme sans recours aux énergies renouvelables et à la récupération des matières premières, aboutirait rapidement à l'épuisement des ressources planétaires.

${ }^{6}$ Ce ne sont pas les plus riches en termes de revenus par habitant, et les États les plus riches ont souvent des populations d'effectifs modestes : le Luxembourg, la Norvège, le Qatar, et d'autres encore.
} 
Cette recherche prend de nouveau appui sur les données statistiques de qualité régulièrement rassemblées ou élaborées par le PNUD, et plus particulièrement sur celles qui ont fait l'objet du dernier rapport publié en $2013^{7}$, dans lequel les données les plus récentes datent de 2011, ainsi que sur les précieux outils et indices mis en place par cette équipe.

\section{Revenus, santé, éducation. Variations selon les États}

Le PNUD utilise des indicateurs ou séries d'indicateurs représentant ces trois domaines et intervenant, chacun pour un tiers, dans la détermination de l'indice de développement humain $(\mathrm{IDH})^{8}$.

1 - Les revenus (Tableau 2).

\begin{tabular}{|c|c|c|c|c|c|c|}
\hline & \multicolumn{3}{|c|}{$\mathrm{RNB} / \mathrm{h}^{1}$} & \multicolumn{3}{|c|}{ Population } \\
\hline & Dollars & Rang $^{2}$ & Décile & Millions & Cumul & $\%$ urbains \\
\hline \multicolumn{7}{|c|}{ États émergents (BRICS) } \\
\hline Russie & 14561 & 53 & $9^{e}$ & 147,8 & - & 73,2 \\
\hline Brésil & 10162 & 77 & $8^{e}$ & 196,7 & 344,5 & 86,9 \\
\hline Afrique du Sud & 9469 & 79 & $8^{e}$ & 50,5 & 395,0 & 62,2 \\
\hline Chine & 7476 & 94 & $5^{e}$ et $6^{e}$ & 1347,6 & 1742,6 & 47,8 \\
\hline Inde & 3468 & 124 & $3^{e}$ et $4^{e}$ & 1241,5 & 2984,1 & 30,3 \\
\hline \multicolumn{7}{|c|}{ Groupe témoin } \\
\hline États-Unis & 43017 & 10 & $10^{e}$ & 313,0 & - & 87,6 \\
\hline Canada & 35166 & 16 & $10^{\mathrm{e}}$ & 34,2 & 347,2 & 80,7 \\
\hline Allemagne & 34854 & 17 & $10^{e}$ & 82,2 & 429,4 & 74,0 \\
\hline Japon & 32295 & 23 & $10^{e}$ & 126,5 & 555,9 & 67,0 \\
\hline Cuba & 5416 & 103 & $5^{e}$ & 11,3 & 567,2 & 75,2 \\
\hline Monde - 187 États & 10082 & - & - & - & - & 50,8 \\
\hline
\end{tabular}

Tableau 2. Revenus et populations des États émergents et du groupe témoin

\footnotetext{
7 L'essor du Sud: le progrès humain dans un monde diversifié, Programme des Nations-Unies pour le développement, 2013.

${ }^{8}$ Toutes les données sont arrondies, à partir des données vraies (données 2011).
} 
L'indicateur principal, qui intervient seul dans l'indice, est le revenu national brut par habitant (RNB/h). Il est revu annuellement et constitue un élément fiable du niveau de vie moyen des populations ${ }^{9}$. On y adjoint parfois d'autres indicateurs, par exemple le taux d'urbanisation.

Parmi les 5 États du BRICS, le RNB/h évolue de 14 600\$ en Russie (53e rang mondial, 148 millions d'habitants - groupe des États très riches du $9^{e}$ décile) à $3500 \$$ en Inde ( $124^{\mathrm{e}}$ rang, 1,24 milliard - État pauvre des $3^{\mathrm{e}}$ et $4^{\mathrm{e}}$ déciles). Les 3 autres États se situent en position plus intermédiaire : le Brésil, 10 200\$ (197 millions) et l'Afrique du Sud, $9500 \$$ (50,5 millions), à quasi égalité des rangs (77 et $79^{\mathrm{e}}$ ), au niveau de la moyenne mondiale (10 100\$) et au $8^{e}$ décile, celui des États riches ; la Chine, enfin, avec $7500 \$(1,35$ milliard), au niveau médian, séparant l'ensemble des humains de la planète en deux parts égales, l'une plus pauvre et l'autre plus riche. Au total et quant aux revenus, il s'agit d'un groupe d'États assez hétérogène, ce qui se confirme lorsqu'on observe le degré d'urbanisation. Deux États restent assez faiblement urbanisés, la Chine à 48 \% et l'Inde à $30 \%$; les 3 autres le sont majoritairement : 62 \% en Afrique du Sud, 73 \% en Russie et $87 \%$ au Brésil.

L'homogénéité des revenus, si l'on excepte Cuba, est plus forte dans le groupe témoin puisqu'on a retenu 4 États du $\mathrm{G} 7$, tous $\mathrm{du} 10^{\mathrm{e}}$ décile et extrêmement riches. Retenons cependant que les États-Unis, 43 000\$ (10 ${ }^{\mathrm{e}}$ rang, 313 millions d'habitants), se détachent assez nettement du groupe compact des 3 autres : Canada, $35200 \$\left(16^{\mathrm{e}} \mathrm{rang}\right.$, 34 millions d'habitants); Allemagne, $34900 \$\left(17^{\mathrm{e}}\right.$ rang, 82 millions) ; Japon, $32300 \$$ (23 rang, 126,5 millions). Cuba, qui compte 11 millions d'habitants, dispose d'un RNB/h beaucoup plus modeste -5 400\$\$, 8 fois moins que les États-Unis-, qui ne le place qu'au $103^{\mathrm{e}}$ rang, aux $5^{\mathrm{e}}$ et $6^{\mathrm{e}}$ déciles, entre la Chine et l'Inde. L'homogénéité concernant les taux d'urbanisation est très forte, et toujours égale ou supérieure aux deux tiers, y compris à Cuba : il n'y a plus d'importantes régions non urbanisées, comme en Chine ou, plus encore, en Inde.

\footnotetext{
${ }^{9}$ Le produit intérieur brut (PIB) définit la richesse annuelle globalement produite.
} 
2 - La santé $\left(\right.$ Tableau $\left.3^{10}\right)$.

L'espérance de vie à la naissance constitue le premier indicateur de vie et santé de la population d'un État. II résume de fait tous les autres et évolue en fonction de leurs évolutions respectives : par exemple, parmi les facteurs d'accroissement de l'espérance de vie à la naissance, le premier concerne la diminution de la mortalité infantile.

Le PNUD l'a bien compris, en privilégiant cet indicateur pour représenter le domaine vie et santé dans la détermination de I'IDH. II en utilise néanmoins d'autres, de façon à compléter l'information : par exemple, l'espérance de vie ajustée à la santé ${ }^{11}$, les taux de non-vaccination des populations (diphtérie, coqueluche et tétanos d'une part, rougeole d'autre part), ceux de mortalité des enfants de moins de 5 ans et ceux de mortalité des adultes, hommes ou femmes, avant l'âge de 60 ans, ainsi que la part de PIB consacrée aux dépenses publiques de santé. Ce sont les indicateurs que nous avons systématiquement retenus. Nous aurions pu en adjoindre d'autres, par exemple le nombre moyen de naissances par femme ou le taux de mortalité infantile avant un an ; ils paraissaient moins essentiels eu égard aux États et populations retenues.

\begin{tabular}{|c|c|c|c|c|c|c|c|c|c|}
\hline & \multirow{3}{*}{$\mathrm{EV}^{1}$} & \multirow{3}{*}{$\mathrm{EV} / \mathrm{S}^{2}$} & & & & \multicolumn{4}{|c|}{ MA $15-60^{5}$} \\
\hline & & & \multicolumn{3}{|c|}{ Vaccinations $^{3}$} & \multirow{2}{*}{$\begin{array}{l}\text { MI av. } \\
5 \text { ans }^{4}\end{array}$} & \multirow[b]{2}{*}{$\mathbf{F}$} & \multirow[b]{2}{*}{ H } & \multirow{2}{*}{$\begin{array}{l}\text { Dép. } \\
\text { Santé }^{6}\end{array}$} \\
\hline & & & DCT & \multicolumn{2}{|c|}{$\mathbf{R}$} & & & & \\
\hline \multicolumn{10}{|c|}{ États émergents (BRICS) } \\
\hline Russie & 68,8 & 60 & & 2 & 2 & 12 & 144 & 391 & 3,5 \\
\hline Brésil & 73,5 & 64 & & 1 & 1 & 21 & 102 & 205 & 3,8 \\
\hline Afrique du Sud & 52,8 & 48 & & 1 & 38 & 62 & 479 & 521 & 4,0 \\
\hline Chine & 73,5 & 66 & & 3 & 6 & 19 & 87 & 142 & 2,7 \\
\hline Inde & 65,4 & 56 & & 4 & 29 & 66 & 169 & 250 & 1,3 \\
\hline \multicolumn{10}{|c|}{ Groupe témoin } \\
\hline États-Unis & 78,5 & 70 & & 5 & 8 & 8 & 78 & 134 & 8,4 \\
\hline Canada & 81,0 & 73 & & 0 & 7 & 6 & 53 & 87 & 8,1 \\
\hline Allemagne & 80,4 & 73 & & 7 & 4 & 4 & 53 & 99 & 9,0 \\
\hline Japon & 83,4 & 76 & & 2 & 6 & 3 & 42 & 86 & 7,8 \\
\hline
\end{tabular}

\footnotetext{
${ }^{10}$ Sur ce tableau figurent, chaque fois qu'elles sont connues, les données mondiales moyennes. Un repère essentiel pour la lecture des autres.

${ }^{11}$ Cet indicateur estime le nombre d'années en moins bonne santé (maladies ou accidents bien définis). L'espérance de vie ajustée à la santé est donc la différence entre l'espérance de vie et ce nombre d'années.
} 


\begin{tabular}{|c|c|c|c|c|c|c|c|c|}
\hline Cuba & 79,1 & 69 & 4 & 4 & 6 & 78 & 120 & 11,2 \\
\hline Monde - 187 États & 69,8 & 61 & 18 & 18 & 58 & 137 & 211 & \\
\hline \multicolumn{9}{|c|}{$\begin{array}{l}{ }^{1} \text { Espérance de vie à la naissance. En années. }{ }^{2} \text { Espérance de vie ajustée à la santé. En années. }{ }^{3}{ }^{4} \text { Pourcentage d'enfants d'un an non vaccinés. DCT : diphtérie, coqueluche, tétanos; R: rougeole. }{ }^{4} \\
\text { Taux de mortalité des enfants de moins de } 5 \text { ans. Pour } 1000 \text { naissances vivantes. }{ }^{5} \text { Taux de } \\
\text { mortalité avant } 60 \text { ans des adultes de } 15 \text { ans, hommes ou femmes. Pour } 1000 .{ }^{6} \text { Dépenses } \\
\text { publiques de santé. Part du PIB (\%). Données } 2009 \text { ou voisines. }\end{array}$} \\
\hline
\end{tabular}

Tableau 3. Indicateurs de vie et santé des États émergents et du groupe témoin

Observons d'abord les données du groupe témoin. Sur tous les indicateurs ou presque, les scores sont meilleurs que les moyennes mondiales : un gain de presque ou plus de 10 ans en espérance de vie et espérance de vie en bonne santé, des taux de vaccination et de mortalité infantile à un chiffre à l'exception d'un cas au Canada (DCT), des taux de mortalité des adultes faibles et très inférieurs aux taux mondiaux (ils sont « pour mille » et sur 45 ans) et des dépenses de santé qui approchent ou dépassent $10 \%$ du PIB. Certes, toutes les données évoluent bien dans le même sens (hormis la vaccination DCT dont il faudrait rechercher l'explication) et confirment bien la valeur de synthèse de l'indicateur espérance de vie. Or, il varie de presque 5 ans, ce qui est considérable, entre les États-Unis, dont les revenus sont les plus élevés, et le Japon, le moins riche des 4 du G7, et celui qui fait un peu mieux que les autres, sur l'ensemble de tous les critères, de surcroît avec une part de PIB légèrement inférieure ; à l'inverse, les États-Unis connaissent des taux de mortalité infantile et des 15-60 ans plus élevés : sans doute des politiques de santé différentes. Le Canada et l'Allemagne, proches quant aux revenus, se rapprochent du Japon. Reste Cuba, qui consacre plus de $11 \%$ de son modeste PIB au budget santé et égale ou dépasse, certes légèrement mais sur tous les critères, les ÉtatsUnis dont le RNB/h est 8 fois plus élevé, approchant l'Allemagne et le Canada, deux États extrêmement riches du $10^{\mathrm{e}}$ décile.

L'hétérogénéité est encore plus grande dans le groupe des États émergents. L'espérance de vie varie de 53 ans en Afrique du Sud à 73,5 ans au Brésil et en Chine, passant à 65 ans en Inde puis à 69 ans, près de la moyenne mondiale, en Russie. Les durées d'espérances de vie en bonne santé sont en assez bonne correspondance : des 
durées en «moins bonne santé » réduites à 5 ans en Afrique du Sud et de 7 à 9 ans dans les autres. Les politiques de vaccinations que recommande l'OMS (moins de 5 \% de non vaccinations) sont appliquées au Brésil, en Chine et en Russie, mais la prévention reste très insuffisante en Inde ou en Afrique du Sud où près à plus du tiers des enfants ne sont pas vaccinés. Les taux de mortalité infantile avant 5 ans sont partout à deux chiffres : 12 \% en Russie, soit 4 fois plus qu'au Japon et un taux qui, dans I'UE, n'apparaît plus qu'en Roumanie, 21 et $19 \%$ au Brésil et, surtout, 62 et $66 \%$, plus que la moyenne mondiale, en Afrique et en Chine. Les taux de mortalité des 15-60 ans, notamment des hommes, restent très élevés : seuls ceux de la Chine, $9 \%$ chez les femmes et $14 \%$ chez les hommes, ou, de façon plus limite, ceux du Brésil, 10 et 20 \%, se comparent avec ceux des États-Unis ou de Cuba ; ceux de la Russie, qui a réalisé des progrès vis-à-vis de la mortalité infantile restent élevés (14\%) pour les femmes et très élevés (39\%) pour les hommes ; ceux de I'Inde, notamment pour les femmes (17\%), un peu moins pour les hommes (25\%), doublent quasiment par rapport à la Chine ; enfin, ceux de l'Afrique du Sud, 48 et 52 \%, plus de 10 fois plus qu'au Japon pour les femmes et 6 fois plus pour les hommes, paraissent invraisemblables. Et les parts de PIB allant de 1,3\% (dans le pays le plus pauvre) à 4 \% (en Afrique du Sud) sont certainement insuffisantes pour des progrès significatifs. Des dépenses de santé représentant 3 à 4 \% du PIB dans les pays les plus riches, des parts qui s'amenuisent notamment en Chine, où le budget santé ne représente plus que 2,7 \% du PIB, et plus encore en Inde où il régresse à $1,3 \%$ de son PIB.

En définitive et pour résumer, les 10 États des deux groupes, en fonction de l'espérance de vie, se classent selon 5 niveaux ${ }^{12}$.

- $\quad$ Espérance très élevée, 80 ans et plus : Canada, Allemagne et Japon, 3 États extrêmement riches, tous du groupe témoin, ayant des résultats assez proches d'autres États comparables, de l'UE par exemple.

- $\quad$ Espérance élevée, 74 à 79 ans : États-Unis, extrêmement riche, Cuba, médian, tous deux du groupe témoin. Le premier obtient des résultats un peu en deçà de son niveau de revenu, le second des résultats très au delà.

\footnotetext{
${ }^{12}$ Ce classement reprend celui qui a été utilisé dans la recherche effectuée à propos de l'UE.
} 
- Espérance moins élevée, 68 à 73 ans : Russie, très riche, et Brésil, riche, l'un et l'autre du groupe témoin, Chine, médian et État émergent. Les résultats de la Russie restent un peu en deçà de ceux qu'on aurait pu attendre, ceux du Brésil au niveau attendu et ceux de la Chine un peu au delà.

- $\quad$ Espérance basse, 60 à 67 ans : Inde, pauvre. La pauvreté est sans doute le facteur principal de ses difficultés.

- $\quad$ Espérance très basse, 53 à 59 ans : Afrique du Sud, État émergent riche. Les revenus, très proches de ceux du Brésil, ne peuvent, à eux seuls, expliquer ces résultats. D'autres causes seront à rechercher.

$$
\text { 3- L'éducation (Tableau } 4^{13} \text { ). }
$$

\begin{tabular}{|c|c|c|c|c|c|c|c|}
\hline & \multirow[b]{2}{*}{ DMS $^{1}$} & \multirow[b]{2}{*}{$\mathrm{TAA}^{2}$} & \multicolumn{4}{|c|}{ Taux brut scol. ${ }^{4}$} & \multirow[b]{2}{*}{$\begin{array}{l}\text { Dép. } \\
\text { Educ. }^{5}\end{array}$} \\
\hline & & & $\mathrm{DAS}^{3}$ & Prim. & Sec. & Sup. & \\
\hline \multicolumn{8}{|c|}{ États émergents (BRICS) } \\
\hline Russie & 9,8 & 99,6 & 14,1 & 96,8 & 84,8 & 77,2 & 4,4 \\
\hline Brésil & 7,2 & 90,0 & 13,8 & 127,5 & 100,8 & 34,4 & 5,7 \\
\hline Afrique du Sud & 8,5 & 88,7 & 13,1 & 101,2 & 93,9 & - & 5,5 \\
\hline Chine & 7,5 & 94,0 & 11,6 & 112,7 & 78,2 & 24,5 & - \\
\hline Inde & 4,4 & 62,8 & 10,3 & 116,9 & 60,0 & 13,5 & 3,1 \\
\hline \multicolumn{8}{|c|}{ Groupe témoin } \\
\hline États-Unis & 12,6 & - & 16,0 & 98,2 & 93,6 & 85,9 & 5,4 \\
\hline Canada & 12,1 & - & 16,0 & 98,4 & 102,2 & 62,3 & 4,8 \\
\hline Allemagne & 12,2 & - & 15,9 & 103,6 & 101,7 & & 4,6 \\
\hline Japon & 11,6 & - & 15,1 & 102,3 & 101,0 & 58,6 & 3,4 \\
\hline Cuba & 9,9 & 99,8 & 17,5 & 99,3 & 93,5 & 67,1 & 14,1 \\
\hline Monde - 187 États & 7,4 & 80,9 & 11,3 & 106,9 & 68,4 & 27,6 & - \\
\hline \multicolumn{8}{|c|}{$\begin{array}{l}{ }^{1} \text { Durée moyenne de scolarisation des personnes de } 25 \text { ans et plus, en nombre d'années. }{ }^{2} \text { Taux } \\
\text { d'alphabétisation des adultes de } 15 \text { ans et plus. Dernière donnée connue entre } 2005 \text { et } \\
2010 .{ }^{3} \text { Durée attendue de scolarisation. En nombre d'années, pour les enfants en âge d'entrer à } \\
\text { l'école l'année considérée. }{ }^{4} \text { Taux brut de scolarisation. Primaire, secondaire et supérieur. } \\
\text { Dernières données connues entre } 2001 \text { et } 2010 .{ }^{5} \text { Dépenses publiques d'éducation. Part du PIB (\%). } \\
\text { Données } 2009 \text { ou voisines. }\end{array}$} \\
\hline
\end{tabular}

Tableau 4. Indicateurs d'éducation des États émergents et de ceux du groupe témoin

\footnotetext{
${ }^{13}$ Sur ce tableau figurent, chaque fois qu'elles sont connues, les données mondiales moyennes. Elles sont un repère essentiel.
} 
L'Organisation des Nations Unies considère que le développement humain dans le domaine de l'éducation ne peut se réaliser que s'il vise à fois les jeunes et les adultes; le PNUD a longtemps utilisé deux indicateurs principaux, l'un évaluant les taux d'alphabétisation des adultes, l'autre les taux de fréquentation des divers segments du système éducatif, du primaire au supérieur. Dorénavant, et c'est le cas pour la période examinée ici, il utilise les durées moyennes de scolarisation des jeunes et des adultes. Pour les adultes, il a retenu la durée moyenne de scolarisation (DMS), en nombre d'années, effectuée par l'ensemble de la population âgée de 25 ans et plus. Pour les jeunes en cours de scolarisation, il s'agit de la durée attendue de scolarisation (DAS), donc un pronostic. DMS et DAS interviennent, à parts égales, dans le calcul de l'indice de développement humain

Le PNUD utilise d'autres indicateurs connexes parmi lesquels nous avons retenu :

- $\quad$ les taux d'alphabétisation des adultes, qui mettent en évidence une très forte concordance avec les DMS ;

- $\quad$ les taux bruts actuels de scolarisation, du primaire au supérieur ;

- comme pour le domaine de la vie et de la santé, les taux de dépenses publiques d'éducation.

Dans le groupe témoin, aucun des 4 États extrêmement riches ne renseigne quant aux taux d'alphabétisation des adultes et Cuba, État médian, indique un taux très proche de $100 \%$ : il n'y a plus, ou pratiquement plus, d'analphabétisme dans ce groupe, même si perdure un taux d'illettrisme difficile à identifier. Les DMS confirment d'ailleurs ce bon niveau d'éducation des adultes : très élevé, autour de 12 ans ou plus aux États-Unis, au Canada, en Allemagne et au Japon, élevé à Cuba. Les données concernant la fréquentation de l'enseignement primaire (de $98 \%$ aux États-Unis et au Canada à plus de $100 \%$ - des doubles inscriptions - au Japon ou en Allemagne) montrent qu'il est totalement généralisé ; la situation est une peu différente en ce qui concerne l'enseignement secondaire : à la fois à Cuba et aux États-Unis, 6 à 7 \% des jeunes, part faible mais non négligeable, continuent d'y échapper. En revanche, et notamment dans 
ces deux États, la fréquentation des enseignements supérieurs est très élevée (plus des deux tiers) aux États-Unis ( 86 \%) et à Cuba 67 \%), élevée (un tiers à moins de deux tiers) au Canada $(62 \%)$ et au Japon $(59 \%)^{14}$. Les durées attendues de scolarisation des jeunes (DAS) se partagent entre le niveau élevé dans lequel se regroupent les 4 États extrêmement riches (Japon, 15 ans, États-Unis, Canada et Allemagne, 16 ans) et le niveau très élevé dans lequel ne figure que Cuba (17 ans) qui rejoint, quelques rares États de la planète, notamment des États d''Europe du Nord. La part de PIB consacrée aux dépenses d'éducation est un peu plus faible au Japon (3\%), plus homogène dans les 3 autres États extrêmement riches et nettement plus élevée à Cuba (14\%)

Dans le groupe des États émergents, la DMS varie de 4 ans (DMS basse) en Inde, durée très inférieure à la DMS moyenne d'environ 7 ans, à 10 ans (DMS élevée) en Russie, valeur équivalant celle de Cuba ; elle est moins élevée ( 8,5 ans) en Afrique du Sud et basse au Brésil et en Chine (7 ans). Les taux d'alphabétisation sont en correspondance avec ces durées dans 4 cas sur 5 : le maximum est observé en Russie (pratiquement $100 \%$ ) et le minimum (63\%) en Inde ; ils sont plus intermédiaires au Brésil (90\%) et en Chine (94\%), mais ils stagnent à $89 \%$ en Afrique du Sud ${ }^{15}$. La situation semble difficile pour I'Inde, d'autant que, si elle a généralisé son enseignement primaire, elle est aussi en retard quant au développement de son enseignement secondaire $(60 \%)$ et, surtout, de son enseignement supérieur (13,5\%) : sur l'ensemble des dimensions, elle figure en catégorie basse. La Russie étonne : elle a certes un potentiel, sa DMS, mais elle n'a pas achevé la généralisation de son enseignement secondaire (85\%), et projette une DAS à 14 ans (catégorie moins élevée) alors que 77 \% de ses jeunes accèdent déjà à l'enseignement supérieur. Les 3 autres États semblent chercher à avancer : ils accroissent la DAS par rapport à la DMS, de plus de $6 \%$ au Brésil et de 4 à $5 \%$ en Chine et Afrique du Sud, et, en même temps, généralisent l'accès à l'enseignement secondaire (100 \% au Brésil, 94 \% en Afrique du Sud et 78 \% en Chine), malgré les inégalités qu'ils connaissent et sur lesquelles nous reviendrons. Les taux d'accès à l'enseignement supérieur, 24,5\% pour la Chine, classée au niveau bas et 34 \% pour le Brésil, classé au niveau moins élevé, semblent en

\footnotetext{
${ }^{14}$ Taux non indiqué par l'Allemagne.

${ }^{15}$ Ce qui ne peut correspondre qu'à une distribution des durées de plus forte variance.
} 
concordance avec ce développement ${ }^{16}$.

En définitive, en tenant compte à la fois de l'éducation des jeunes et de celle des adultes, les 10 États des deux groupes se répartissent ainsi :

- niveau global très élevé : États-Unis, extrêmement riche, Cuba, médian, groupe témoin. Le premier obtient des résultats correspondant à son niveau de revenu, le second des résultats très au delà ;

- niveau global élevé : Canada, Allemagne et Japon, 3 États extrêmement riches du groupe témoin ; Russie, État émergent très riche. Les 3 premiers enregistrent des résultats un peu en deçà de leurs revenus, le $4^{\mathrm{e}}$ des résultats en concordance ;

- $\quad$ niveau global moins élevé : 2 États riches du groupe émergent, Afrique et Brésil. Résultats en bonne concordance avec les revenus ;

- $\quad$ niveau global bas. La Chine, État médian, se situe un peu au delà de ses revenus, l'Inde, pauvre, en concordance avec les siens.

\section{4 - Revenus, santé et éducation}

Ces 3 domaines, pris successivement 2 à 2, revenus et santé d'une part, revenus et éducation d'autre part, ont mis en évidence des corrélations assez fréquentes, mais aussi de nettes discordances. En prenant en même temps en compte les 3 domaines, on peut retenir la classification suivante:

- 2 États du groupe des pays émergents apparaissent en concordance forte. Le Brésil, État riche, obtient des résultats de niveau moins élevé, tant en ce qui concerne le domaine santé qu'en ce qui concerne le domaine éducation. L'Inde, État pauvre, y observe des résultats bas ;

- 6 États sont en concordance plus atténuée, produisant des résultats un peu différents, mais cependant voisins, selon les domaines. Parmi les États émergents, la Russie, très riche, classée au niveau élevé dans le domaine

\footnotetext{
${ }^{16}$ Taux non indiqué par l'Afrique du Sud. Compte tenu des autres renseignements fournis, elle est, comme le Brésil, classée au niveau moins élevé.
} 
éducation, ne se classe qu'au niveau moins élevé dans le domaine santé ; à l'inverse, la Chine, État médian reste au niveau bas dans le domaine éducation, mais atteint le niveau moins élevé dans celui de la santé. De même, dans le groupe témoin, parmi les 4 États extrêmement riches, deux cas inverses sont observés : les États Unis, en cohérence avec leurs revenus, se situent au niveau très élevé en éducation, mais au niveau élevé dans le domaine santé ; et les 3 autres, Canada, Allemagne et Japon inversent le schéma : résultats de niveau très élevé dans le domaine santé, de niveau élevé dans le domaine éducation. Sans doute des choix politiques différents, et des investissements financiers, qu'il serait intéressant d'étudier ;

- 2 États marquent des discordances nettement plus accentuées et qui méritent d'être relevées. L'Afrique du Sud, État riche, produit des résultats de niveau moins élevé dans le domaine de l'éducation (comme le Brésil) en bonne concordance avec ses revenus, mais des résultats, notamment quant à l'espérance de vie, qui la placent encore au niveau très bas, parmi les États les plus en difficulté de la planète, et qui restent le produit d'une histoire durant laquelle les hommes, pour reprendre la belle expression qu'utilise le PNUD, n'étaient que des «intrants nécessaires à l'augmentation de la production ». Au contraire, l'exemple de Cuba montre une réussite étonnante malgré des revenus médians dérisoires, réussite rejoignant celle des États-Unis, tant dans le domaine de la santé que dans celui de l'éducation.

Certes, le revenu est un moyen, et un moyen indispensable, nous l'avons observé notamment avec l'Inde qui connaît de sérieuses difficultés de développement. Mais, plus que le revenu lui-même, c'est aussi son mode d'utilisation et de répartition à l'intérieur des populations, et bien évidemment les politiques qui sont menées, pour les dimensions essentielles du développement humain. 


\section{Inégalités et indices. Un pas vers la conclusion}

Toutes les données présentées dans les pages précédentes constituent de bons indicateurs du développement humain. Et elles ont, entre elles, une bonne cohérence. Elles permettent un portrait un portrait de chacun des États, et par conséquent des comparaisons, entre ces États ou avec d'autres pour lesquels on irait rechercher les mêmes types de données. Je ne peux qu'encourager le lecteur à cette démarche ; comparer par exemple son pays à un autre, ou à plusieurs.

Un indice est généralement un résumé, sous forme quantifiée, effectué à partir d'indicateurs estimés pertinents. C'est le cas des indices produits par le PNUD, qui indique précisément ce qu'il résume, et comment il le résume.

Le PNUD ${ }^{17}$ a élaboré une série d'indices concernant le développement humain. Nous en utiliserons deux : l'indice proprement dit (IDH) et l'indice ajusté aux inégalités (IDHI). Il a, par ailleurs, très récemment, mis au point un judicieux indice d'inégalité de genre (IIG) que nous présenterons également. Enfin, nous utiliserons le coefficient de $\mathrm{Gini}^{18}$, qui mesure les inégalités de distribution des revenus. Ces indices sont regroupés dans le tableau 4. Ainsi verrons-nous comment se construisent, et se superposent les inégalités de développent humain que connaissent les populations de ces États.

\begin{tabular}{|c|c|c|c|c|c|c|c|c|c|}
\hline & \multicolumn{2}{|c|}{$\begin{array}{l}\text { IDH }^{1} \\
2011\end{array}$} & \multicolumn{2}{|c|}{$\begin{array}{l}I^{I D H I^{2}} \\
\text { ajusté } \\
2011\end{array}$} & \multicolumn{2}{|c|}{$I I G^{3}$} & $\mathrm{RQR}^{4}$ & \multicolumn{2}{|c|}{ G Gini ${ }^{5}$} \\
\hline \multicolumn{10}{|c|}{ États émergents (BRICS) } \\
\hline Russie & .755 & 66 & .670 & 11,3 & .338 & 59 & - & .310 & 16 \\
\hline Brésil & .718 & 84 & .519 & 27,7 & .449 & 80 & 17,6 & .539 & - \\
\hline Afrique du Sud & .619 & 123 & - & - & .490 & 94 & 20,2 & .578 & 33 \\
\hline Chine & .687 & 101 & .534 & 22,3 & .209 & 35 & 8,4 & .415 & 18 \\
\hline Inde & .547 & 134 & .392 & 28,3 & .617 & 129 & 5,6 & .325 & - \\
\hline \multicolumn{10}{|c|}{ Groupe témoin } \\
\hline États-Unis & .910 & 4 & .771 & 15,3 & .288 & 47 & 8,5 & .408 & 16 \\
\hline
\end{tabular}

\footnotetext{
${ }^{17}$ Données et indices sont aisément accessibles et téléchargeables :

http://hdr.undp.org/fr/content/rapport-sur-le-développement-humain-2013

${ }^{18}$ Ces 3 indices ont été utilisés dans notre dernier ouvrage traitant de l'UE (op. cité, 2014).
} 


\begin{tabular}{|c|c|c|c|c|c|c|c|c|c|}
\hline Canada & .908 & 6 & .829 & 8,7 & .140 & 20 & 5,5 & .326 & - \\
\hline Allemagne & .905 & 9 & .842 & 6,9 & .085 & 7 & 4,9 & .330 & - \\
\hline Japon & .901 & 12 & - & - & .123 & 14 & 3,4 & ,249 & 4 \\
\hline Cuba & .776 & 51 & - & - & $\cdot 337$ & 5 & - & - & - \\
\hline Monde. 187 États & .682 & - & .525 & 23,0 & . 492 & & & & \\
\hline \multicolumn{10}{|c|}{$\begin{array}{l}{ }^{1} \text { Indice de développement humain. A gauche, valeur (entre o et } 1 \text { ); à droite, rang (sur } 187 \text { ). }{ }^{2} \text { Indice } \\
\text { de développement humain ajusté aux inégalités. A gauche, indice corrigé en fonction des } \\
\text { inégalités propres à l'État considéré; à droite, pourcentage de correction par rapport à l'IDH. }{ }^{3} \\
\text { Indice d'inégalité de genre. Données } 2011 . \text { A gauche, valeur de l'indice (entre o et } 1 \text { ); à droite, rang } \\
\text { (sur } 146 \text { États). }{ }^{3} \text { Rapport des quintiles de revenu. Valeur du rapport entre le premier et le } \\
\text { cinquième. }{ }^{5} \text { Coefficient G de Gini. A gauche, valeur (entre o et } 1 \text { ); à droite, valeur du rapport entre } \\
\text { le premier et le dixième décile. . }\end{array}$} \\
\hline
\end{tabular}

Tableau 5. Les indices d'inégalités. États émergents et groupe témoin

1 - Le développement humain et ses indices

« Le concept du développement humain accorde la priorité aux fins plutôt qu'aux moyens du développement et du progrès. Le véritable objectif du développement devrait en effet consister à créer un environnement propice à une vie meilleure en termes de durée, de santé et de créativité ».

Publication PNUD, 2011

L'IDH renseigne sur la potentialité des États, à partir des 3 domaines retenus (revenus, santé et éducation) et à parts égales. Il définit ce que devrait réaliser chaque État, qu'il classe en fonction de ce score : parmi les 10 États, les États-Unis ont le meilleur potentiel, 0,910, et occupent le rang 4 au niveau mondial ; I'Inde est dernière (rang 134), avec un score de 0,547 .

Compte tenu des inégalités relevées par le PNUD (sur les mêmes critères pour tous les États), celui-ci produit l'indice ajusté aux inégalités (IDHI), l'indice réel, toujours inférieur à l'IDH (il n'y a pas d'égalité parfaite), et calcule, en pourcentages, les pertes liées aux inégalités : les États-Unis ont perdu $15 \%$, mais l'Inde $28 \%$.

Ces pertes constituent un véritable gâchis : $23 \%$ au niveau mondial, près d'un 
quart du bien le plus précieux. Dans notre corpus, sont surtout épargnés le Canada, $9 \%$ et I'Allemagne, $7 \%{ }^{19}$; mais les États émergents sont les principales victimes (des pertes de l'ordre de 28 \% pour le Brésil, et sans doute plus pour l'Afrique du Sud...), parce qu'ils comptent encore beaucoup de pauvres. Le seul moyen de les faire régresser, c'est de diminuer les inégalités, pour réduire la pauvreté, voire l'éradiquer.

\section{2 - Les inégalités de genre}

L'indice (IIG) est construit sur un modèle voisin comprenant également 3 domaines - la santé, l'autonomisation et le marché de l'emploi -, chacun intervenant pour un tiers dans le calcul de l'indice final. Le choix des indicateurs semble assez représentatif et pertinent : concernant la santé, le taux de mortalité maternelle et le taux de fécondation des adolescentes ; l'autonomisation, à partir des proportions femmeshommes des niveaux d'instruction (secondaire ou supérieur) ${ }^{20}$, et des répartitions femmes-hommes dans les assemblées parlementaires; enfin, l'accès à l'emploi est calculé à partir des proportions femmes-hommes dans la population active ${ }^{21}$.

Le meilleur score est obtenu par l'Allemagne, avec un indice de 0,085 , très proche de 0 , et le $7^{\mathrm{e}}$ rang mondial : il y a quelques rares pays dans lesquels les inégalités de genre ont pratiquement disparu ; à l'inverse, elles sont très fortes en Inde (0,617 et $\left.129^{\mathrm{e}} \mathrm{rang}\right)$. Elles restent faibles au Canada et au Japon, moyennes en Chine, élevées aux États-Unis, à Cuba et en Russie, très élevées en Afrique du Sud et au Brésil.

Mais, à eux seuls, les indices ne suffisent pas à qualifier ces inégalités. Il est nécessaire d'observer la manière dont elles se construisent, et les résultats selon les divers indicateurs. Certes, deux indices nettement différents mettent en évidence des nettes différences de degré quant aux inégalités de genre : par exemple, les États-Unis sont moins inégalitaires que la Russie ; mais seule l'observation des indicateurs met en évidence que la différence est essentiellement liée au partage de l'emploi, moins

\footnotetext{
${ }^{19}$ Au niveau mondial, les pertes minimales sont de $4 \%$ : Suède et République tchèque.

${ }^{20}$ Les situations sont parfois favorables aux femmes.

${ }^{21}$ Le lecteur intéressé par cette analyse peut se reporter à notre dernier ouvrage paru en 2014, dans lequel un chapitre est consacré à ces inégalités et à ces États.
} 
inégalitaire aux États-Unis qu'en Russie. Surtout, l'égalité ou la proximité des indices (ici, Canada et Japon) peut masquer, par compensation, des différences opposées : ces deux États sont égalitaires, mais le Japon, plus égalitaire que le Canada en ce qui concerne la santé, l'est, au contraire, beaucoup moins en ce qui concerne l'accès à l'emploi. Un bel exemple des nécessaires complémentarités entre qualitatif et quantitatif.

Ces inégalités, dans de nombreux pays, restent très fortes voire s'accroissent, notamment en période de crise et de chômage et lorsque la pauvreté croît. Et ce sont toujours des inégalités surajoutées, par rapport aux autres inégalités : elles sont, par exemple, notamment dans les pays pauvres, en très forte corrélation avec les pertes de développement humain.

3 - Les inégalités de répartition des revenus

Le coefficient de Gini ${ }^{22}$ est un très bon indice. Le principe est simple : il consiste à répartir les revenus par tranches de population, le plus souvent de 10 \% (décilage), des plus pauvres aux plus riches, et d'évaluer l'évolution des revenus selon ces tranches ; si l'égalité était parfaite, chaque tranche recevrait la même part, et si l'inégalité était parfaite, les 9 premières ne recevraient rien, la dixième tout. En théorie, il varie de 0 (égalité maximale) à 1 (inégalité maximale); en pratique, entre les États de la planète, il varie entre 0,2 (inégalités très faibles) et 0,7 (inégalités très fortes), avec une médiane de 0,4 .

Il est renseigné par tous les États, sauf Cuba ; et il varie de .249 au Japon (État très égalitaire) à .578 en Afrique du Sud (État très inégalitaire). Certains coefficients sont accompagnés d'un nombre : 4 pour le Japon, 33 pour l'Afrique du Sud. Ce nombre est très important car il exprime le rapport entre les parts des déciles extrêmes :

- $\quad$ au Japon, les revenus des 10 \% les plus riches, ne sont, en moyenne, que 4 fois supérieurs à ceux des $10 \%$ les plus pauvres ;

- $\quad$ en Afrique du Sud, le rapport est de 1 à 33, ce qui explique la pauvreté d'une

\footnotetext{
${ }^{22}$ Il doit son nom au statisticien, démographe et sociologue italien Corrado Gini (1884-1965). Ce coefficient a été décrit et utilisé dans nos deux ouvrages cités.
} 
grande partie de sa population.

Le Japon, plus riche, atténue la pauvreté ; l'Afrique du Sud, moins riche, la renforce encore.

Ce rapport entre les déciles n'est pas toujours indiqué. C'est pourquoi nous avons fait figurer un rapport entre les quintiles de revenu (RQR), moins précis mais permettant, par interpolation, une estimation du rapport entre les déciles ${ }^{23}$.

Parmi les États émergents, le Brésil $(G=.539)$ est inégalitaire, et les $10 \%$ les plus pauvres reçoivent des revenus 29 fois inférieurs aux $10 \%$ les plus riches. Vient ensuite la Chine, dans laquelle les inégalités de revenus sont médianes $(G=.415)$, avec un rapport encore élevé, 18 ; plus proches des États égalitaires, I'Inde et la Russie avec des coefficients voisins (.325 et .310) restent dans la catégorie médiane, et le rapport entre les déciles extrêmes reste plus élevé en Russie (16) que le rapport estimé en Inde (12).

Dans le groupe témoin, avec le Japon déjà cité, se classe l'Allemagne (.283, rapport de 7), autre Etat très inégalitaire. Le Canada, en léger décalage, et classé égalitaire ( $G$ = .326, rapport estimé à 10) se situe au niveau de la Russie ou de l'Inde, mais avec des revenus à partager nettement supérieurs à ceux de la Russie et sans commune mesure avec ceux de l'Inde. Enfin, on trouve les Etats-Unis ( $G=.408$, rapport de 16), Etat classé au rang médian, et à égalité avec la Chine.

Hormis les Etats-Unis, ceux qui ont renseigné sont moins que les Etats émergents qui continuent de subir le poids de leur passé, comme l'Afrique du Sud notamment. Cuba n'a pas renseigné, mais ses résultats montrent au moins que sa population a bénéficié de redistributions ayant permis son niveau de développement dans les domaines de la santé et de l'éducation.

Enfin, et chacun peut le vérifier, les inégalités de répartition des revenus sont souvent corrélées avec les inégalités de genre, mais aussi avec les pertes de développement humain qu'elles entraînent.

\footnotetext{
${ }^{23}$ L'interpolation est le calcul d'une " $4^{\text {e }}$ proportionnelle ». Prenons l'exemple du Brésil par rapport à I'Afrique du Sud: le rapport entre les déciles est tel que $x / 33=17,6 / 20,2$, soit 29 . Et ainsi de suite.
} 


\section{Pour conclure}

Que l'on regarde l'Union européenne ou les Etats émergents, les conclusions sont quasi identiques : entre les Etats et à l'intérieur des Etats, le développement humain est freiné par les inégalités de toutes sortes et de tout genre, qui continuent de s'accroître plutôt qu'elles ne se résorbent, dans une course sans fin aux profits et à la croissance insensée d'un produit intérieur brut devenant une fin alors qu'il n'est qu'un moyen.

Les pertes de développement humain constituent un gâchis invraisemblable, notamment dans les pays pauvres comme l'Inde, mais aussi dans les Etats émergents comme l'Afrique du Sud ou le Brésil, atteignant bien évidemment les populations les plus fragiles et les plus démunies; et même, certes de façon plus atténuée, dans la première puissance économique mondiale, les Etats-Unis.

La réponse est simple et se lit à chaque page : favoriser le développement humain, celui de tous les humains, en réduisant les inégalités les plus criantes, toutes les inégalités, et notamment celles qui réduisent des pans entiers de populations à la précarité et la grande pauvreté. Partout, et les médias nous le rappellent, la colère gronde, parmi les plus démunis et tout particulièrement dans les pays émergents ; et la jeunesse s'impatiente...

Le concept de développement humain développé par Amartya Sen et repris dans l'ensemble des travaux du PNUD sera, j'en suis convaincu, le concept majeur du $21^{\mathrm{e}}$ siècle. Et il faut qu'il le soit, car il est une réponse aux errements des économistes libéraux qui tentent encore de croire - ou de nous faire croire - que l'accroissement du PIB profite à tous, alors qu'il ne profite qu'à une infime minorité, au détriment de tous les autres et d'une planète pillée, saccagée et sérieusement menacée.

Ces inégalités, qui se juxtaposent et, surtout, se surajoutent atteignent notamment l'éducation, principal moteur du développement humain. Aujourd'hui, sans doute plus encore qu'au moment où elle a été élaborée, voilà un demi-siècle, la théorie la plus explicative demeure celle de l'héritage culturel et de la reproduction sociale élaborée 
par Pierre Bourdieu et Jean-Claude Passeron ${ }^{24}$; et elle le demeurera tant que les inégalités resteront fortes : aujourd'hui, en bien des cas, le facteur «méritocratique », pour reprendre la terminologie de Raymond Boudon $^{25}$, ne suffit plus pour inverser le poids du facteur de « dominance ».

Mais les théories ne sont pas éternelles, sinon elles ne seraient que des dogmes. Et elles sont d'autant plus tenaces qu'elles ont été constituées sur des bases solides : c'est le cas de la théorie de la reproduction; et elle se renforcera si les inégalités s'accroissent. A l'inverse, elle s'affaiblira si les inégalités reculent: la reproduction sociale n'est pas une fatalité, mais une simple conséquence de l’organisation sociale.

Ce sont les inégalités qu'il faut faire reculer. C'est le « tout PIB » qu'il faut revoir et considérer comme un moyen à mieux maîtriser, et en aucun cas une fin. Au service d'un objectif essentiel, le développement humain, dont le concept reste sans doute encore à approfondir, et dont la mesure des progrès serait un indicateur au moins aussi pertinent que le PIB. Une société sans doute imparfaite, dont certains Etats se sont déjà davantage rapprochés, en Europe du Nord par exemple, mais aussi d'autres, parmi les anciens Etats socialistes. Une société dans laquelle l'éducation, les savoirs et les compétences auraient, enfin, une vraie place.

\footnotetext{
${ }^{24}$ Bourdieu, P., Passeron, J.-C., Les héritiers, Ed. de Minuit, 1964 ; La reproduction, Paris, Ed. de Minuit, 1970. Les héritiers vient d'être traduit par deux de mes collègues et ami(e)s du Brésil. Je tiens à saluer cette traduction qui me semble très réussie.

${ }^{25}$ Boudon, R., L'inégalité des chances. Paris, 1973.
} 


\section{Références bibliographies}

BOUDON, Raymond. L’inégalité des chances. Paris: Armand Colin, 1973.

BOURDIEU, Pierre; - -PASSERON, Jean-Claude. La reproduction, éléments pour une théorie du système d'enseignement. Paris: Éditions de Minuit, 1970.

BOURDIEU, Pierre; - e-PASSERON, Jean-Claude. Les héritiers: - Lles étudiants et la culture. Paris: Ed. de Minuit, 1964.

BOURDIEU, Pierre; - ePASSERON, Jean-Claude.Os herdeiros:-. Oos estudantes e a cultura. Florianópolis: Editora da UFSC, 2014.

LANGOUËT, Gabriel. (Préface de Roger Establet). Les inégalités entre États et populations de la planète, Trop, c'est trop !. Préface de Roger Establet.--Paris: L'Harmattan, 2011.

LANGOUËT, Gabriel. Les inégalités dans l'Union européenne et ailleurs : . Eet si on osait? Paris: L'Harmattan, 2014.

PNUD- PROGRAMME DES NATIONS UNIES POUR LE DEVELOPPEMENT. Rapport sur le développement humain 2013. $\leftarrow$ l'essor du Sud: le progrès humain dans un monde diversifié. New York: PNUD, 2013. Disponível em:

<http://www.st.undp.org/content/dam/sao_tome_and_principe/docs/Rapport\%20STP/und p_st_HDR2013French.pdf >. Acesso em junho de 2014 .

PNUD. PROGRAMME DES NATIONS UNIES POUR LE DEVELOPPEMENT. Rapport sur le développement humain 2011. Durabilité et Équité: Uun Meilleur Avenir pour Tous. New York: PNUD, 2011. Disponível em:

<http://hdr.undp.org/sites/default/files/hdr_2011_fr_complete.pdf >. Acesso em maio de 2014.

Universidade do Estado de Santa Catarina - UDESC Programa de Pós-Graduação em Educação - PPGE Revista Linhas

Volume 15 - Número 29 - Ano 2014 revistalinhas@gmail.com 